Horizons philosophiques

\title{
Sujet(s) à interprétation(s) : sur la relative transparence d'une photographie positiviste
}

\section{Jean Lauzon}

Volume 7, numéro 2, printemps 1997

L’héritage de l'herméneutique

URI : https://id.erudit.org/iderudit/801048ar

DOI : https://doi.org/10.7202/801048ar

Aller au sommaire du numéro

Éditeur(s)

Collège Édouard-Montpetit

ISSN

1181-9227 (imprimé)

1920-2954 (numérique)

Découvrir la revue

\section{Citer cet article}

Lauzon, J. (1997). Sujet(s) à interprétation(s) : sur la relative transparence d'une photographie positiviste. Horizons philosophiques, 7(2), 127-139.

https://doi.org/10.7202/801048ar d'utilisation que vous pouvez consulter en ligne.

https://apropos.erudit.org/fr/usagers/politique-dutilisation/ 


\title{
SUJET(S) À INTERPRÉTATION(S) : SUR LA RELATIVE TRANSPARENCE D'UNE PHOTOGRAPHIE POSITIVISTE
}

\author{
"Nous regardons la photographie [...] \\ comme s'il s'agissait réellement de l'objet [...] \\ qui y est dépeint. II pourrait en être autrement». \\ Wittgenstein
}

Au XIXe siècle, le savoir devait passer par le moule de l'observation d'où l'on recueillait les faits du monde, établissant ainsi ses lois générales de fonctionnement. Attitude positiviste et vérité semblaient ainsi vouloir aisément s'associer. C'était comme si le monde se donnait lui-même sans intermédiaire, comme si l'observation elle-même ne pouvait trahir ce qu'elle observait, comme si les outils étaient neutres, sans opinion ou, pour ainsi dire, transparents.

Au XIXe siècle, on invente la photographie (rendue publique en 1839). On mise alors sur sa «capacité de permettre à la nature de se reproduire elle-même» (Daguerre), sans médiation. Voilà un instrument sans lequel on ne peut plus désormais prétendre connaître quelque chose (Zola). L'interprétation photographique semble vouloir se limiter à la constatation.

Aliquid stat pro aliquo, disaient les Anciens. Voilà donc que l'image photographique vaut pour sa transparence absolue, son identité avec le monde qu'elle communique comme un fait établi. La photographie ne se distingue pas de ce qu'elle photographie. Elle ne vaut pas pour quelque chose d'autre, elle est cette autre chose. Le monde s'enroule en quelque sorte sur luimême (Foucault, 1966), comme si ce nouvel outil fonctionnait de fait sous le mode adamique alors que le «langage était un signe des choses absolument certain et transparent» (Foucault, 1966). Le positivisme photographique ressuscite l'ancienne confusion entre herméneutique et sémiologie. II y a pourtant longtemps que le monde et ses signes cherchent à se séparer. 
Au siècle de la photographie, les choses se présentent comme si le divorce tardait à s'actualiser.

À bien regarder toutefois, plusieurs pratiques photographiques du XIXe siècle présentent certains signes d'une transparence pour le moins incertaine. II n'y a qu'à noter la pluralité des procédures de production pour s'autoriser à suggérer la pertinence d'autant de protocoles interprétatifs. Malgré sa propension à l'indiscernabilité des présumés identiques, le positivisme photographique ne résiste guère à l'analyse de l'utilisation de son langage. Le monde et ses images partagent sans doute bien des points communs, mais il n'en demeure pas moins que l'outil de traduction puisse aussi s'entendre, selon la formule bien connue, comme un instrument de trahison.

La pratique photographique propose une singulière façon de voir le monde: tout en le regardant elle s'y colle, peinant de fait à s'en détacher. Ce détachement est toutefois nécessaire puisque sans lui on ne saurait établir la différence entre la chose et sa présentation planaire. II y aurait donc à la fois identité et différence.

Nous proposons de regarder d'un peu plus près certaines pratiques photographiques du siècle positiviste, d'examiner si les différents dispositifs mis en place présentent quelque invariance - question de méthode - et de voir en quoi ces différents protocoles modifient en quelque sorte cette ambition à la transparence du langage. Au delà de l'énoncé, il y aura l'énonciation et dans le sens des images se réfléchira le fait désormais sémantique de cette énonciation (d'après Récanati, 1979). Le monde ne s'enroulera plus sur lui-même. On réservera alors cette acrobatie au langage photographique luimême, d'autant plus que les images ne seront plus seules. Elles feront aussi l'objet de catégories, ces façons de classer le monde et ses représentations, qui elles-mêmes s'enrouleront indéfiniment sur leurs interprétations et usages divers.

Afin de montrer leur diversité, nous passerons rapidement en revue quelques-uns des protocoles photographiques du $\mathrm{XIXe}$ siècle. Nous traiterons particulièrement d'un cas singulier, celui de l'anthropométrie judiciaire d'Alphonse Bertillon, là où 
l'expression «sujet(s) à interprétation(s)» pourra devenir polysémique, à la fois pour les choses photographiées, pour la chose "photographie» et pour tout ce que l'on pourra en dire ou en faire. En fait, nous tenterons de voir comment, en fonction de quelques modalités, on peut interpréter les différents dispositifs mis en place et ainsi, peut-être, faire échec à la transparence, en l'opacifiant.

\section{De quelques cas.}

Question de méthode, et de catégories, il n'est pas question de recenser toutes les pratiques photographiques du XIXe siècle. Nous retiendrons quelques dispositifs mis en place en fonction d'un seul objet : l'être humain. Le portrait a constitué une bonne partie des activités photographiques du siècle dernier et bien qu'il s'agisse toujours du même objet d'investigation, car c'est de volonté de savoir et de connaissance qu'il s'agit, les multiples protocoles d'appréhension de la personne humaine font état d'autant d'intérêts pour ce seul sujet. Bien que la photographie ait très souvent été utilisée pour son caractère transparent, du moins à ce moment, il semble que ce distinctif ne lui sied pas toujours, et qu'il faille donc le relativiser en fonction des points de vue: d'abord dans la perspective des intentions, ensuite en relation avec le dispositif privilégié.

\subsection{De quelques spectres :}

a) L'optogramme - Procédé pour le moins fantaisiste pour un regard actuel (1997), il s'agissait de retrouver sur le fond des yeux d'une personne assassinée le portrait de son meurtrier. En photographiant la rétine, elle-même assimilée à une sorte de camera obscura, il semblait plausible de pouvoir y retrouver l'image de l'assassin, captée au moment même de l'exécution de son crime. Bien que l'efficacité du procédé, fondée sur la croyance en la transparence d'une double image, celle de l'œil et du dispositif photographique, n'ait pas connu une fortune considérable, il n'en a pas moins suscité un intérêt avoué (Dubois, 1986). Nous sommes en 1870. 
b) Psychicônes et périsprit - L'âme humaine aura soulevé bien des discussions. Pouvoir la photographier aurait au moins le mérite de démontrer son existence. Un homme de science respecté en son temps l'aura fait. Ainsi le docteur Hippolyte Baraduc a-t-il photographié la lumière de l'âme. Nommée «psychicône" lorsqu'effectuée sans caméra, cette apparition pourra aussi s'appeler »aura“, telle l'aura de la tristesse, par exemple.

Pour d'autres, il s'agira de photographier le lien qui unit le corps avec l'âme. Appelée "périsprit» (Thuillier, 1980), cette relation mi-matière et mi-esprit, peut être aperçue par l'appareil photographique. Des images existent pour en témoigner, dans le cadre de pratiques spirites dont le représentant connu s'appelait Allan Kardec (Thuillier,1980). Nous sommes dans la seconde moitié du XIXe siècle. L'enregistrement photographique demeure toujours le garant de l'authenticité des faits observés.

c) Photographie composite - II s'agit, par exemple, de faire poser successivement sur la même plaque photographique tous les membres d'une même famille (Villodre, 1985). Le "portrait» ainsi obtenu donne les lignes types de la famille par l'impression sur la plaque sensible des seuls traits communs. II va sans dire qu'il s'agit d'un portrait en quelque sorte fictif. On peut ainsi produire l'image d'une famille, d'une race ou encore de la femme idéale en superposant les images des prétendantes à un concours de beauté (Villodre, 1985). D'abord récréative, cette pratique aura donné lieu à des ambitions au caractère un peu moins ludique. Ainsi l'image composite du peuple anglais imaginée par l'anthropologiste Galton (Moles, 1995) peut-elle s'inscrire au sein d'une pratique eugénique visant à l'amélioration de la race par l'élimination des sujets considérés non-conformes au type idéal ${ }^{1}$.

\subsection{De quelques catégories:}

a) L'hystérie - À l'hôpital de la Salpêtrière de Paris, en 1875 , le docteur Jean-Martin Charcot, neurologue, commence à photographier le corps de ses malades en convulsion. Invitant

1. Galton est notamment l'auteur d'une enquête sur l'hérédité du génie... 
un photographe extérieur à l'établissement pour faire le travail, celui-ci arrivait souvent après que la crise soit terminée. Les premières tentatives de fixer les différents états des malades atteints d'hystérie s'avèrent ainsi peu fructueuses. On crée donc un atelier de photographie à l'hôpital, qui sera confié, à partir de 1882, à Albert Londe.

Pour le docteur Charcot, l'observation photographique, garante de vérité, ne visait pas à considérer les individus dans leur singularité. La pellicule photographique avait plutôt le mandat, pour ainsi dire, d'enregistrer des images à partir desquelles un type général pourrait être établi, et qui rendrait compte de l'hystérie (Carroy, 1994). Le dispositif était fondé sur un souci de classification manifeste à partir duquel la personne était peu à peu délaissée au profit de quelque loi scientifique générale. La technique de l'image composite a aussi été utilisée dans l'atelier de Londe, où l'on a superposé plusieurs photographies d'autant de gens différents afin d'établir le portrait-type de chacune des affections considérées en soi (Rouillé, 1986) ${ }^{2}$.

Ce genre de pratique correspond tout à fait à l'attitude positiviste voulant qu'à partir d'observations factuelles singulières, on puisse en arriver à établir des lois scientifiques générales et universelles. À partir d'occurrences, on cherche à établir un type, à savoir une catégorie particulière soit, dans le cas qui nous occupe, celle de l'hystérie.

b) Photographie et locomotion - Autre dispositif visant à l'établissement de lois générales à partir de cas particuliers, où l'individu est donc utilisé comme matière à induction, celui du médecin physiologiste Étienne-Jules Marey : la chronophotographie. Aussi utilisée par Albert Londe, cette technique permet d'enregistrer sur une même plaque photographique plusieurs

2. La photographie n'a pas été la seule pratique visant à rendre compte de types généraux. Le cas du peintre Théodore Géricault est à ce titre tout aussi représentatif, puisqu'il réalise, au début du siècle, une série de portraits d'aliénés voulant aussi montrer ce qui pourrait constituer l'archétype de quelques affections mentales. Par exemple, le tableau intitulé Aliénée monomane de l'envie (1822-23). 
étapes d'un même mouvement ${ }^{3}$. Marey invente un dispositif à obturateur rotatif, en plus d'un fusil photographique, qui permet de capter sur une seule image toutes sortes de mouvements, allant de la locomotion humaine au vol des oiseaux, en passant par le trajet d'une balle rebondissante. II s'agit d'une sorte de photographie composite sauf qu'au lieu de superposition, on parle ici de juxtaposition sur une même émulsion de plusieurs étapes successives d'un même déplacement. On mise sur le potentiel analytique et l'efficacité scientifique (Rouillé, 1986) des résultats obtenus. Encore ici, on vise à l'établissement de lois générales de fonctionnement à partir de l'observation de cas précis transmutés en étalons de référence. À partir de la série, se crée en quelque sorte sui generis un prototype fictif.

c) L'anthropométrie judiciaire - Si certaines pratiques photographiques médicales et fonctionnelles avaient comme but de déterminer un type général à partir de cas particuliers, en établissant une moyenne, il en va tout autrement pour l'anthropométrie judiciaire. Faisant figures d'exception, si on oublie le cas de l'optogramme pour ainsi dire pas très convaincant, les protocoles photographiques mis en place par Alphonse Bertillon pour reconnaître les criminels, avaient comme objectif essentiel de pouvoir les identifier précisément comme individu. Les différences primaient donc sur les moyennes trans-individuelles qui avaient comme effet de détruire ces disparités, nécessaires pour Bertillon.

Alphonse Bertillon a mis au point un système «pour assurer la reconnaissance des repris de justice» (Bertillon, 1913), dont on connaît encore aujourd'hui quelques prolongements dans les fameux portraits de face et de profil des présumés criminels. Non seulement s'agissait-il de faire le portrait des prévenus, mais encore fallait-il procéder à plusieurs mensurations précises, couplées d'images photographiques, d'une série de traits

3. On connaît aussi l'utilisation de techniques analogues par le photographe Muybridge qui, en 1878 , met au point un système permettant la prise de photographies séquentielles sur plusieurs images différentes. Ainsi sera-t-il célèbre pour avoir montrer le déplacement du cheval au galop, notamment quant à la position des pattes de l'animal au cours des différentes phases de son déplacement. Son procédé serait à l'origine du développement de la "photofinish", qui permet de départager les vainqueurs des courses de chevaux. 
anatomiques que nous partageons tous selon des dimensions qui varient cependant d'un individu à l'autre. Ainsi mesurait-on la hauteur de la tête, sa largeur, la longueur du medius, du pied, de la coudée, la taille, la longueur de l'auriculaire, de l'oreille, et considérait-on également la couleur des yeux ${ }^{4}$.

Bertillon a élaboré un répertoire classé trichotomiquement, en fonction de petites, moyennes ou grandes longueurs, qui contenait 90000 fiches signalétiques en 1890. Pour procéder à une identification il suffisait, en quelque sorte, d'éliminer au fur et à mesure de la recherche les mesures non-correspondantes et, par division par trois quant aux données pré-enregistrées, en arriver «d'élimination en élimination au paquet final qui devra contenir le signalement recherché si, bien entendu, la personne arrêtée a déjà été condamnée et mesurée antérieurement» (Bertillon, 1913) ${ }^{5}$.

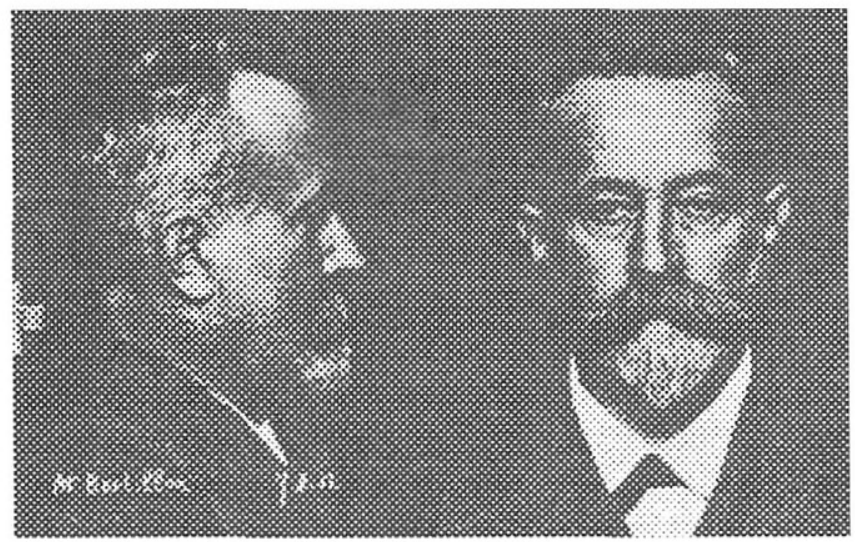

A. Bertillon, autoportrait signalétique, 1912.

4. Le père d'Alphonse Bertillon était anthropologiste. Alphonse l'avait assisté dans ses recherches, notamment quant à l'établissement de mensurations destinées à la description et l'identification des races par l'établissement de moyennes. II devait se détacher des méthodes mises de l'avant par son père pour précisément se consacrer à l'identification des individus dans leurs singularités. Par ailleurs le frère d'Alphonse Bertillon, Jacques Bertillon, était démographe et utilisait également les statistiques dans son travail. Mesures et calculs semblaient garantes de vérité.

5. Le répertoire de Bertillon ne comptait que des représentations d'hommes. Pour les femmes, "beaucoup moins nombreuses" (1913) Bertillon estimait que la photographie seule pouvait suffire à leur identification. 
Le système de mesures mis au point par Bertillon ne constitue donc pas une «incapacité de la photographie» (Rouillé,1986) à pouvoir identifier certains individus, mais s'avère de fait un outil complémentaire permettant de retracer la personne recherchée à travers un classement qui prend figure de langage, et dont le code doit être connu pour assurer son efficacité. II s'agit moins de mettre en relation une personne et son image, mais bien de réussir à naviguer à travers un répertoire chiffré de ces mêmes images afin de pouvoir retrouver celle que l'on recherche et ainsi, éventuellement, l'individu correspondant. Ce n'est donc pas la problématique de la transparence photographique qui apparaît prépondérante, mais bien l'éventuelle transparence du système de classement régissant l'interprétation.

C'est ainsi que les pratiques photographiques entendues seules ne peuvent pas signifier beaucoup 6 , sauf à les relier à certains systèmes interprétatifs que nous nous proposons maintenant de développer quelque peu.

\section{De quelques invariances.}

Des trois premiers cas considérés, on peut sans doute affirmer qu'ils constituent des chimères d'ailleurs sans suite quant aux objectifs escomptés. Pas très scientifiques, malgré les prétentions du moment, et davantage lunatiques, au mieux ludiques, ces recherches spectrales n'en ont pas moins constitué des pratiques photographiques considérées sérieuses aux yeux des contemporains. Fondé sur la transparence présumée de l'image photographique, ce caractère sérieux s'appuyait aussi sur l'esprit du temps qui voulait qu'aux faits observés corresponde la réalité des choses. L'équation réalité et photographie s'imposait d'emblée. C'était sans compter les protocoles d'interprétation.

Des trois autres cas, on admettra qu'ils connurent une fortune plus enviable. Par exemple, des études de locomotion de Marey naîtront notamment le principe du cinématographe, les

6. Au point même où Alphonse Bertillon aurait songé à éliminer la photographie de ses protocoles d'identification. A ce sujet, voir Christian Phéline, L'image accusatrice,1985, p. 133. 
Nu descendant l'escalier de Duchamp, les études de mouvement des Futuristes Bragaglia — photodynamique - , ou encore les recherches sur ordinateur des mouvements d'athlètes visant à l'amélioration de leurs performances sportives. De Bertillon, dont on a abandonné le système de mensurations, on a retenu l'idée de pouvoir identifier un individu en enregistrant ce qu'il a de singulier, en l'occurrence aujourd'hui : son héritage génétique. À l'instar des empreintes digitales et contrairement aux propositions de Londe ou de Marey, ce système vise la personne comme entité singulière et non comme représentant un type général 7 .

L'invariant qui demeure dans tous les dispositifs vus jusqu'ici, c'est que le modèle est toujours un sujet humain. Ce qui varie toutefois, c'est le statut du modèle : type ou occurrence? $\mathrm{Ce}$ qui ne varie pas également, c'est le caractère photographique (transparent?) des dispositifs utilisés; ce qui varie aussi et surtout, c'est le protocole mis en place pour l'interprétation des données enregistrées, notamment par systèmes de classement.

\subsection{Statut du modèle :}

a) Type ou occurrence - Le type sera défini comme ce qui représente une règle générale, qui s'applique à tous les individus d'une même catégorie. II sera induit de l'observation de plusieurs cas particuliers dont on retiendra les caractères communs à partir desquels un modèle unique, nécessairement fictif, sera construit. C'est le cas des "psychicônes" pour l'aura de la tristesse, par exemple, ou encore des "hystériques" photographiés par Londe, de même que celui de la locomotion animale de Marey.

L'occurrence sera entendue comme ce qui n'appartient qu'à un seul individu, ce qui permettra de le singulariser au sein d'un groupe. Le cas de l'anthropométrie judiciaire nous semble à ce

7. On peut par ailleurs considérer aujourd'hui que cette singularité peut prendre certaines allures de loi générale. Certaines recherches actuelles tendent à identifier chez certains individus des gènes directement liés à certaines affections précises; par exemple le gène du "tueur en série». En aucun cas cependant cette loi générale ne saurait dépendre de la seule apparence "photographique" des sujets considérés. 
titre exemplaire ${ }^{8}$. Plutôt que de faire disparaître les différences individuelles au profit d'une reconnaissance catégorielle, l'anthropométrie judiciaire visait le contraire pour ainsi estomper les alibis de la reconnaissance et ainsi réduire, au point de l'annihiler, tout risque d'erreur d'interprétation, celle-ci visant précisément à l'identification d'un seul individu au sein d'une vaste collectivité.

Bien que dans chaque cas le modèle soit l'objet d'un traitement particulier qui nous éloigne, par exemple, du portrait classique en studio, tous ne peuvent être considérés comme faisant partie d'une même catégorie procédurale. Dans cette perspective, considérer les dispositifs privilégiés selon leur appartenance à une stratégie d'investigation relevant du type ou de l'occurrence nous apparaît révélateur. On évite ainsi d'affirmer que dans les cas de Londe, Marey et Bertillon, on ait affaire à un renoncement de l'individu (Rouillé, 1986) au profit d'un dispositif et d'un système interprétatif qui les transcendent.

Pour l'anthropométrie judiciaire, c'est précisément du contraire dont il s'agit. Au sein d'un groupe, on cherche l'individu, alors que pour les autres stratégies mentionnées, le protocole s'inverse: à partir des individus, on cherche à établir un groupe à savoir, par exemple, celui des hystériques.

b) Singulier ou pluriel - Pour Bertillon, il n'apparaît pas aisément qu'ait existé une chose telle que le criminel type que l'on définirait à partir d'une moyenne des apparences offertes par la somme de plusieurs photographies et mesures d'individus différents. C'est la singularité qui l'intéressait, pas la moyenne. Cependant, son répertoire se conjugue au pluriel. Les choses se présentent comme s'il avait reconstitué une grande société de criminels fichés, à l'image de la collectivité visée, et qu'à partir de ce groupe ainsi constitué l'identification individuelle puisse s'actualiser.

8. La pratique de l'optogramme, malgré son caractère farfelu, semble aussi répondre au caractère de l'occurrence. Dans ces deux cas, il s'agit de rendre compte d'une singularité, d'une situation nécessairement unique. Si des types peuvent être considérés, ils seront par ailleurs traduits dans des termes autres que ceux de la représentation photographique, par exemple socioéconomiques. 
C'est tout le contraire pour les travaux de Londe ou de Marey, où la constitution du groupe entendu comme catégorie se présente comme point d'arrivée et non comme point de départ. Pour Bertillon, il s'agit d'une stratégie d'inférence propre à la déduction, alors que pour ses contemporains, l'induction est manifestement privilégiée.

Du singulier au pluriel, ou du pluriel au singulier, voilà donc un protocole d'interprétation aux polarités qui s'inversent radicalement. Ce qui semble alors offrir quelques points d'interrogations ne réside plus dans une problématique interrogeant la transparence ou l'opacité des techniques photographiques utilisées, mais bien plutôt dans l'utilisation des images au sein de modalités interprétatives différentes.

\subsection{L'image ou son protocole :}

On ne discute plus beaucoup aujourd'hui de la pertinence de savoir si, oui ou non, il y a relation nécessaire entre le modèle et sa photographie. Cette contiguïté principielle, indiciaire, est admise. La problématique porte davantage sur l'écart qui doit tout aussi nécessairemnent s'installer entre le moment de la prise d'une photographie et l'apparition de l'image à proprement parler. Sans cet écart, il ne pourrait pas non plus y avoir de photographie. De fait, c'est au sein de cette ambivalence, et non d'une ambiguïté, que toute question relative à la photographie devrait, nous semble-t-il, être considérée, peut-être sous la double notion, dès lors constitutive, de transparence et d'opacité.

Dans plusieurs de ses pratiques photographiques, le XIXe siècle positiviste entendait la photo comme absolument transparente. C'est la valeur principielle d'indexicalité qui prévalait, jumelée avec la certitude que l'observation stricte des faits suffisait à rendre compte d'un réel en quelque sorte préexistant. Herméneutique davantage que sémiotique, cette attitude se fonde sur la croyance en l'existence d'une réalité qu'il suffit de regarder attentivement pour dissiper tout ce qui pourrait de prime abord y apparaître sibyllin. C'était sans compter à la fois les protocoles d'observation et les systèmes mis en place pour questionner ce qui était observé et qui doivent aussi être questionnés à leur tour, pour ainsi dire ad infinitum. 
Ce qu'il faudrait interroger ${ }^{9}$, ce sont les protocoles d'interprétation mis en place pour rendre compte des images. Ce faisant, on pourrait en arriver à cette constatation que d'un écart à l'autre : du modèle à son image ainsi que de l'image au système, le réel devient ce que l'on en fait. On pourrait alors éviter de se satisfaire de simples observations, comme si la signification du réel nous pré-existait et que nous devions nous contenter de la constater. En se reconnaissant comme auteurs essentiels du sens que nous prêtons à la réalité, nous devenons ainsi davantage responsables de ce que nous croyons savoir. Rien n'est fourni d'avance et le monde ne peut plus être transparent. Face à lui c'est l'opacité du langage qui s'affiche, et les choses se présentent finalement comme représentations.

En guise de conclusion

Construits davantage que donnés, le monde et ses images font alors l'objet d'autres appréciations et il s'agit alors moins d'interroger la présumée réalité que les langages - souvent arbitraires - qui nous la servent.

Plutôt alors que de croire à un monde qui s'enroule sur luimême dans la pure transparence des choses, c'est le langage lui-même - photographique ou autre - qui se prêtera à ce périlleux exercice qui consiste à se placer constamment en position de "différance" avec un réel qui, finalement, n'en demande peut-être pas davantage!

Jean Lauzon

Université du Québec à Montréal

9. Le $X X^{e}$ siècle a abondamment discuté de l'indexicalité comme valeur essentielle de la photographie, faisant en quelque sorte pencher la balance du côté de l'écart - à quelques exceptions près - qui s'impose nécessairement entre le modèle et son image. C'est toute la question, très ancienne par ailleurs, de la mimêsis, réactualisée sous quelques nouvelles modalités avec la photographie. 


\section{BIBLIOGRAPHIE}

BERTILLON, Alphonse, «Identification-anthropométrie judiciaire», Le Monde et la Science, Paris : Librairie Schwarz \& Cie, 1913, vol. 3, p. 809-818.

CARROY, Jacqueline, "Hypnose et hystérie à la fin du XIXe siècle», Les cahiers de Science \& Vie, Paris : hors-série no 22, août 1994, pp. 818.

DAGUERRE, Jacques Louis Mandé, “Daguerréotype», Classic Essays on Photography, edited by Alan Trachtenberg, New Haven, Conn. (États-Unis), Leete's Island Books, 1980, p. 11-13.

DUBOIS, Philippe, "Le corps et ses fantômes", La Recherche photographique, Paris : Presses Universitaires de Vincennes, no 1, octobre 1986, p. 41-50.

FOUCAULT, Michel, Les mots et les choses, Gallimard, Coll. Tel,1966, $400 \mathrm{p}$.

FRIZOT, Michel, Histoire de voir- le medium des temps modernes (18801939), Paris : Photo Poche, 1989, 143 p.

KEIM, Jean-A., Histoire de la photographie, Paris : P.U.F., "Que sais-je?", 1979, $126 \mathrm{p}$.

MOLES, A. Abraham, Les sciences de l'imprécis, Paris : Points/Seuil, 1995, $360 \mathrm{p}$.

PHÉLINE, Christian, L'image accusatrice, Paris : Les Cahiers de la Photographie, no 17, 1985, 169 p. 111.

RÉCANATI, François, La transparence et l'énonciation, Paris : Seuil, 1979, $215 \mathrm{p}$.

ROUILLÉ, André, "Au-delà du principe physiognomonique», La Recherche photographique, Paris : Presses Universitaires de Vincennes, no 1, octobre 1986, p. 51-55.

SONTAG, Susan, La photographie, Paris, essai/Seuil, 1979, 223 p.

THUILLIER, Pierre, «Évolutionnisme et spiritisme : le cas Wallace», Le petit savant illustré, Paris : Seuil Science ouverte, 1980, p. 51-57.

VILLODRE, Nicolas, «Les récréations photographiques à la fin du XIXe siècle", Photographies, Paris : Fernand Hazan, no 8, septembre 1985, p. 106-109. 